\title{
MR-Based Real Time Path Planning for Cardiac Operations with Transapical Access
}

\author{
Erol Yeniaras ${ }^{1}$, Nikhil V. Navkar ${ }^{1,2}$, Ahmet E. Sonmez ${ }^{1}$, Dipan J. Shah ${ }^{3}$, \\ Zhigang Deng ${ }^{2}$, and Nikolaos V. Tsekos ${ }^{1}$ \\ ${ }^{1}$ Medical Robotics Lab \\ ${ }^{2}$ Computer Graphics and Interactive Media Lab, \\ Department of Computer Science, University of Houston, Houston, TX 77004, USA \\ ${ }^{3}$ Methodist DeBakey Heart \& Vascular Center, Houston, TX 77030, USA \\ \{yeniaras, nvnavkar, aesonmez, ntsekos, zdeng\} @cs.uh.edu, \\ djshahatmhs.org
}

\begin{abstract}
Minimally invasive surgeries (MIS) have been perpetually evolving due to their potential high impact on improving patient management and overall cost effectiveness. Currently, MIS are further strengthened by the incorporation of magnetic resonance imaging (MRI) for amended visualization and high precision. Motivated by the fact that real-time MRI is emerging as a feasible modality especially for guiding interventions and surgeries in the beating heart; in this paper we introduce a real-time path planning algorithm for intracardiac procedures. Our approach creates a volumetric safety zone inside a beating heart and updates it on-the-fly using real-time MRI during the deployment of a robotic device. In order to prove the concept and assess the feasibility of the introduced method, a realistic operational scenario of transapical aortic valve replacement in a beating heart is chosen as the virtual case study.
\end{abstract}

Keywords: Real time MRI, Image Guided Surgeries, and Beating Heart.

\section{Introduction}

Contemporary improvements in the field of medical robotics, and a series of successful clinical applications, have led to the emergence of interventional robots by the clinical and technical community. The inclusion of real-time image guidance in robotic-assisted interventions may further elevate the field by offering improved information-rich visualization, as well as option of assessing the tissue before, during and after a procedure [1]. Considering the challenges associated with the continuous cardiac motion, real-time image guidance can provide a number of benefits especially for robot-assisted surgeries in a beating heart [2, 3].

Among the emerging clinical paradigms in the area of minimally invasive procedures in a beating heart is magnetic resonance imaging (MRI) guided prosthetic aortic valve implantation via transapical access; a procedure that has been demonstrated manually [4] and with robotic assistance [2]. For such off-pump procedures, an important factor is the efficacy of using images to assess the dynamic environment during operation. One effective method is the extraction of dynamic 
access corridors from images [5]. MRI was selected by numerous investigators for its high soft-tissue contrast, absence of ionizing radiation, and inherent robustness and versatility [4, 5]. With the current state-of-the-art MRI, dynamic images can be collected at a rate of 40-50 ms/image. Practically, only a single imaging plane can be collected with such a high, for MRI standards, rate. Thus the question is how a 3D corridor inside the beating heart can be updated using a single plane.

To address this issue, in this work, we evaluate a method that combines preoperative multislice dynamic MRI (i.e., cine MRI) with single-slice real-time MRI to update an access corridor from the apex to the aortic annulus. Cine MRI is used to generate a preoperative 3D corridor in the left ventricle (LV) which is updated on-thefly by registering it onto the intraoperative real-time MR images. The method was assessed for accuracy of the corridor registration and simulated for the deployment of a virtual robot for 12 subjects.

\section{Methodology}

In a typical transapical scenario, the robotic manipulator enters $\mathrm{LV}$ via a trocar affixed at the apex, $\mathrm{T}(\mathrm{t})$; and maneuvers toward the targeted center of aortic annulus, $\mathrm{A}(\mathrm{t})$ as depicted in Fig. 1. Preliminary analyses of the cine data from 12 healthy volunteers indicated that LV can be transversed with a cylindrical corridor and the deployment path from $\mathrm{T}(\mathrm{t})$ to $\mathrm{A}(\mathrm{t})$ is not a straight line. For a precise orthogonal approach to aortic annulus, a dynamic bending point, B ( $t$ ), near the base of LV is needed. The characteristics of the corridor, as well as the aortic annulus diameter, coronary ostial anatomy and apical entrance point were determined from cine MRI, whereas real-time MRI was used to update the corridor and follow the operation.

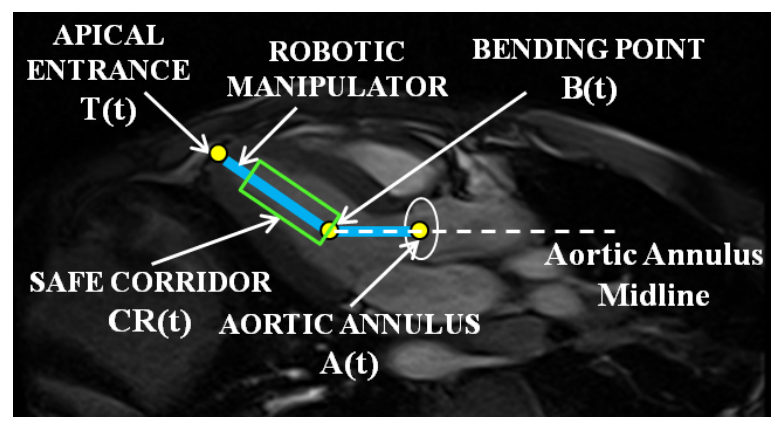

Fig. 1. A long axis MR image shows a typical transapical approach to a beating heart at time $t$

\subsection{Preoperative Planning}

For any given cardiac phase (i.e., at time frame t), the access corridor CR(t) is defined as the largest cylindrical volume that lies along LV from the apex toward the base of the heart. Then, an appropriate-sized surgical device should be able to deploy inside $\mathrm{CR}(\mathrm{t})$ from $\mathrm{T}(\mathrm{t})$ to $\mathrm{A}(\mathrm{t})$ without colliding or injuring the endocardium, papillary muscles or chordae tendinae. The cylindrical corridor was generated from cine 
datasets $(n=12)$ collected with a true fast imaging, steady-state precession pulse sequences (TrueFISP) with TR/TE $=2.3 \mathrm{~ms} / 1.4 \mathrm{~ms}$, flip angle $=80^{\circ}$, slice thickness $=$ $6.0 \mathrm{~mm}$, and acquisition matrix $=224 \times 256$. Each dataset included 19 short axes (SA) and 5 long axes (LA) slices, capturing heart motion with 25 frames over a complete cardiac cycle. In order to determine the transient positions of the corridor and the deployment points (i.e., $\mathrm{T}(\mathrm{t}), \mathrm{B}(\mathrm{t})$, and $\mathrm{A}(\mathrm{t})$ ), SA and LA images were segmented using a region-growing algorithm based on Insight Toolkit (ITK) filters to extract the apex, LV and aortic annulus. As shown in Fig. 2(a) and 2(b), to realistically model the corridor, papillary muscles and chordae tendinae were also considered. For every single heart phase $\mathrm{t}(\mathrm{t}=1$ to 25$), \mathrm{CR}(\mathrm{t})$ is constructed as follows:

1. The SA slices with visible blood pool are determined by checking the inside surface areas of LV segmentations, i.e., selecting the non-zero ones as in Fig. 2(b);

2. LV segmentation contours of these SA slices are projected onto a virtual plane along their common orthogonal axis to find their intersection polygon $\left(\mathrm{INP}_{\mathrm{T}}\right)$ by $2 \mathrm{D}$ polygon clipping. This projection is based on the fact that SA slices are parallel to each other and collected with the same field of view;

3. The largest circle $\left(\mathrm{S}_{\mathrm{T}}\right)$ that fits into $\mathrm{INP}_{\mathrm{T}}$ is determined. Since a circle can be created with a center point and a radius, let`s define $C_{T}$ as the Center of $S_{T}$ and, $R_{T}$ as the Radius of $S_{T}$. Then the centroid of $\mathrm{INP}_{\mathrm{T}}$ is chosen as the center of $\mathrm{S}_{\mathrm{T}}$, shown in Eq. (1) where $\mathrm{N}$ is the number of edges of $\mathrm{INP}_{\mathrm{T}}$ :

$$
\mathrm{C}_{\mathrm{T}}(\mathrm{x}, \mathrm{y})=\frac{1}{\mathrm{~N}} \sum_{\mathrm{i}=1}^{\mathrm{N}} \mathrm{INP}_{\mathrm{T}}\left(\mathrm{x}_{\mathrm{i}}, \mathrm{y}_{\mathrm{i}}\right)
$$

Thus, $\mathrm{R}_{\mathrm{T}}$ can be safely defined as "the minimum distance from $\mathrm{C}_{\mathrm{T}}$ to the edges of $\mathrm{INP}_{\mathrm{T}}$ " as formulated in Eq. (2):

$$
\mathrm{R}_{\mathrm{T}}=\min _{1 \ldots \mathrm{N}}\left(\left\|\mathrm{C}_{\mathrm{T}}(\mathrm{x}, \mathrm{y})-\mathrm{INP}_{\mathrm{T}}\left(\mathrm{x}_{\mathrm{i}}, \mathrm{y}_{\mathrm{i}}\right)\right\|\right)
$$

4. Finally, $S_{\mathrm{T}}$ is stretched from the apex to the base of the heart, through all SA slices to generate a circular straight access corridor as shown in Fig. 2(c).

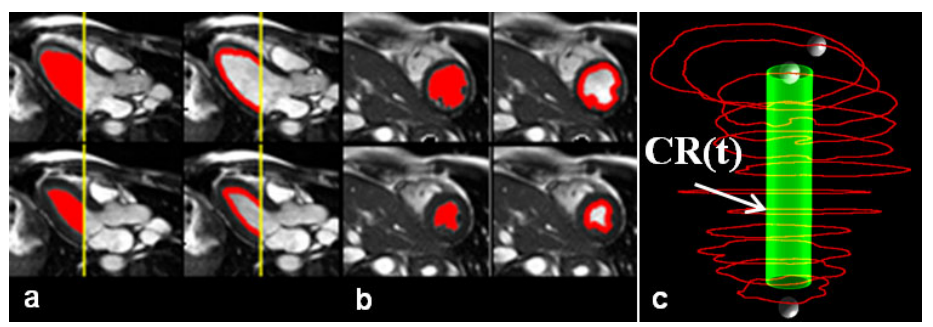

Fig. 2. Selected segmentations of LA (a), SA (b) slices from diastole and systole phases highlight LV blood pool and boundaries, and a sample corridor with endocardial contours (c)

Since a unique 3D access corridor is generated for every single heart phase, CR(t) is a 4D dynamic entity defined for a full heart cycle consisting of 25 time frames. The final step of preoperative planning is to set the initial positions of deployment points as follows: (1) $\mathrm{T}(\mathrm{t})$ is selected manually by a cardiovascular surgeon as the tip of the LV (the apex) on the central LA slice (and verified computationally that it belongs to 
a SA slice that shows only myocardium with no LV blood pool); (2) A(t) is determined from the segmentation contours of two LA and one SA slices that include the aortic valve annulus at the level of aortic valve leaflets. Note that, the initial selection of the exact target point is also made by the surgeon (or cardiologist); (3) $\mathrm{B}(\mathrm{t})$ is assigned as the intersection of the aortic annulus midline and the top face of the safe corridor initially (as also depicted in Fig. 1).

\subsection{Intraoperative Guidance}

In this phase, the access corridor and deployment points, generated preoperatively for a complete heart cycle, are updated on-the-fly. Intraoperative guidance is based on continuous real-time acquisitions of a central LA slice taken from the very same healthy volunteers $(n=12)$ but this time spanning 30 full heart-beats with breathing (TrueFISP parallel imaging with effective repetition time $=48.4 \mathrm{~ms}$, TE $=0.95 \mathrm{~ms}$, alpha $=65^{\circ}$, slice thickness $=6.0 \mathrm{~mm}$, acquisition matrix $=160 \times 66$ ). After comprehensive analyses of MRI data and different imaging planes, we observed that heart mainly translates along and rotates around its long axis without significant outof-plane motion with respect to the real-time LA slice under consideration. Moreover, breathing adds an extra vertical motility with respect to the MR table, as denoted with $\mathrm{R}$ in Fig. 3, which can also be followed on the same LA slice effectively. To the end, we choose this single real-time LA slice for intraoperative guidance.

The most challenging task is to register the preoperative corridor onto the real-time LA slice on-the-fly during the operation. This is done via two major steps: (1) Determine the heart phase in which the real-time slice was collected (and thus match it with the corresponding corridor); (2) Adjust the position and orientation of this corridor to account for heart motion due to respiration, arrhythmias, etc.

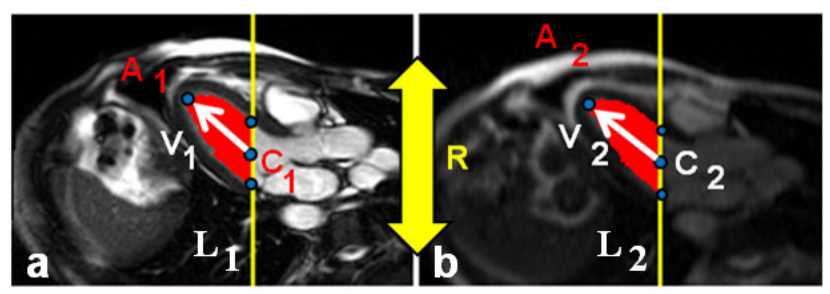

Fig. 3. Illustration of LA segmentations depicting the blood-pool area, R: respiratory motion; $A_{\mathrm{J}}$ : Apex points; $\mathrm{C}_{\mathrm{J}}$ : Midpoints; $\mathrm{L}_{\mathrm{J}}$ : Vertical lines, $\mathrm{V}_{\mathrm{J}}: \mathrm{C}_{\mathrm{J}} \rightarrow \mathrm{A}_{\mathrm{J}} \mathrm{LV}$ directional vectors for nonreal-time and real-time LA slices respectively; $\mathrm{J}=1$ and 2

First, LV is segmented in the real-time LA image using the same region-growing algorithm and area of the blood-pool is calculated. Then, this area is compared to each of the 25 preoperative LV areas of the same LA slice that is extracted with the same parameters, to find the closest. This comparison is done by a conservative approach, i.e., selecting the closest one with the minimum size to guarantee the aforementioned safety criterion. Once it is found, the corridor corresponding to this heart phase is selected as the one to be registered to the real-time LA slice. Fig. 3 shows a segmented cine (non real-time) LA slice and its real-time counterpart respectively. 
Next, the corridor needs to be correctly positioned onto the real-time slice. To achieve that, a vertical line $\left(\mathrm{L}_{2}\right)$ crossing the base of the heart is defined. This line is defined such that segmentation includes most of LV blood pool but not the aortic valve. Then, the intersection points of this line to the endocardial wall are determined and their midpoint $\left(\mathrm{C}_{2}\right)$ is calculated as shown in Fig. 3. The same operation is performed for the corresponding preoperative image to compute $\mathrm{L}_{1}$ and $\mathrm{C}_{1}$, and thus we can compare $C_{1}$ with $C_{2}$. It should be noted that, $A_{1}$ and $A_{2}$ denote the apex points, while $V_{1}$ and $V_{2}$ are directional vectors for the LVs.

Then, the relative displacement between $\mathrm{C}_{1}$ and $\mathrm{C}_{2}$ is calculated and applied to the top-center point of the corridor, $\mathrm{P}_{11}$, to find $\mathrm{P}_{21}$ as shown in Fig.4 (a). To adjust the orientation, the angle between $\mathrm{V}_{1}$ and $\mathrm{V}_{2}$ is calculated and applied to the direction vector of the preoperative corridor $\mathrm{V}_{1 \mathrm{C}}$ to find $\mathrm{V}_{2 \mathrm{C}}$. Finally, the resultant corridor is registered to its new position as depicted in Fig.4 (b) and it is ready for the robotic manipulator. The above process is highly efficient and real-time (i.e., all the computing takes less than $48.4 \mathrm{~ms}$, which is less then effective repetition time).

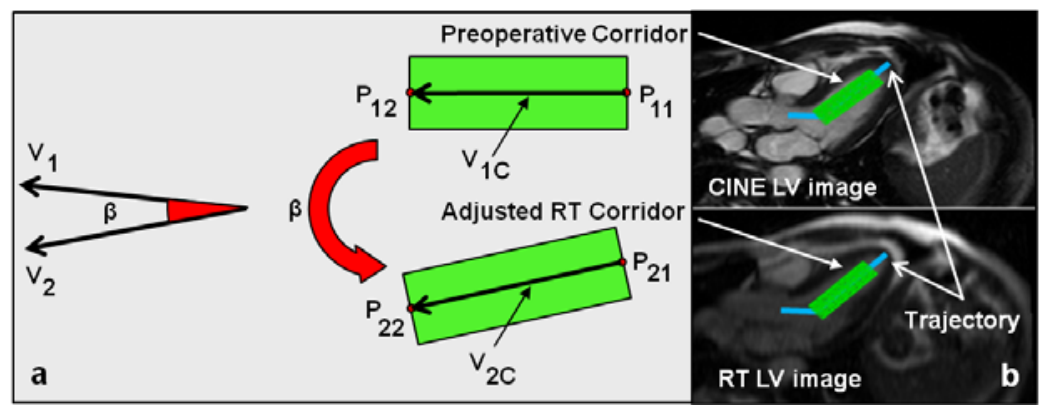

Fig. 4. (a) Corridor registration using vectors; and (b) the registered trajectory on LA slices

Finally, initial deployment points are tracked during the (virtual) operation using an efficient $2 \mathrm{D}$ fast-tissue-tracking algorithm [6]. In an in vivo scenario, the target and the bending points can be tracked with such an algorithm whereas appropriate MRI methods should provide the position of the apex point, $\mathrm{T}(\mathrm{t})$ in real-time (e.g., miniature RF coil beacons on the trocar [7]).

\subsection{Experimental Studies}

The proposed method was tested for the registration accuracy of the corridor onto the real-time MRI. Specifically, the registered corridors were compared to the groundtruths that were created by manually locating them onto their correct positions in all the real-time images for 30 full heart cycles $(n=12)$. Fig. 5 shows three examples of corridor positioning errors.

We also simulated the deployment of a six degrees-of-freedom (DOF) RRPRRP (R: Rotational joint, P: Prismatic joint) virtual robot inside the registered corridor to assess the possible collisions with the endocardium or the aortic walls. The proximal end of the robot was assumed to be attached to $\mathrm{T}(\mathrm{t})$ with a continuous actuation to follow the motion of the apex and Denavit-Hartenberg convention was used in inverse 
kinematics to deploy the robot along the corridor. The inputs for robot control were the dynamic coordinates of deployment points as well as the initial conditions specified by the operator, e.g., the time frame when the robot initiates its maneuvering, and whether and for how long it may hold a certain position along its path. Motion of the virtual robot entails the following three steps: deployment of the first link from the apex toward $\mathrm{B}(\mathrm{t})$, extension of the second link toward $\mathrm{A}(\mathrm{t})$, and the holding of the position. Since the device must stay inside $\mathrm{CR}(\mathrm{t})$, turn can occur before or after the initial $\mathrm{B}(\mathrm{t})$ provided that the first section remains in the corridor and the tip heads to the center of aortic annulus. During the maneuvering process, the robot control supplies the values of the updated DOFs for each time instance. Robot deployment was visually simulated along with the surgical field using OpenGL.

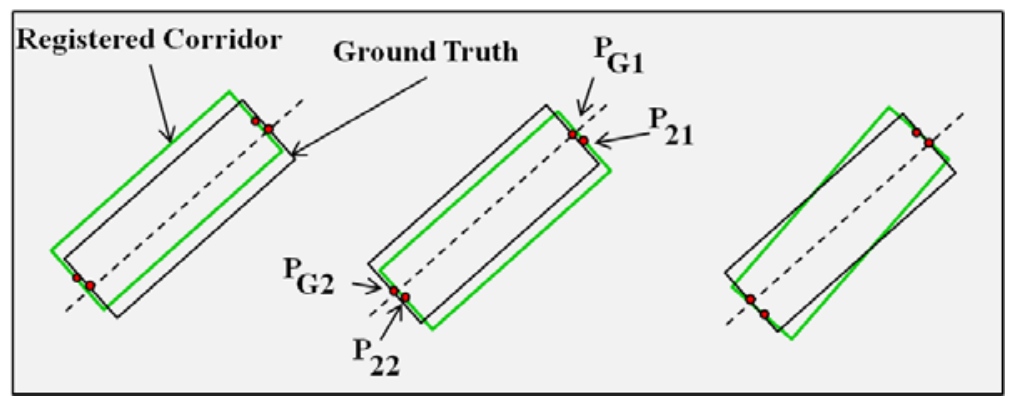

Fig. 5. Registered points: $P_{21}, P_{22}$ and ground-truth: $P_{G 1}, P_{G 2}$ for three different samples

\section{Results and Discussion}

Application of the method on all preoperative cine sets $(n=12)$ demonstrated that a dynamic cylindrical corridor can be defined and tracked for safe deployment inside the LV of the beating heart. The average base diameter of this corridor for 12 subjects was $9 \mathrm{~mm}$ in systole and $22 \mathrm{~mm}$ in diastole. In regard to the code for on-the-fly processing of the real-time MRI, corridor selection was practically error-free as a reflection of the conservative approach in selecting them (i.e., the minimal size). The average distal error for the starting point of the corridor $\left(\mathrm{P}_{21}\right)$ was $1.3 \mathrm{~mm}$ while it was $2.0 \mathrm{~mm}$ for the ending point $\left(\mathrm{P}_{22}\right)$ as formulated in Eq. (3) and listed in Table 1.

$$
\varepsilon_{\mathrm{S}}=\left\|\mathrm{P}_{21}-\mathrm{P}_{\mathrm{G} 1}\right\| ; \varepsilon_{\mathrm{E}}=\left\|\mathrm{P}_{22}-\mathrm{P}_{\mathrm{G} 2}\right\|
$$

This difference is mainly caused by the fact that the ending point is nearer to the apex which is the most dynamic point of a heart, and the starting point is nearer to the aortic annulus which undergoes a relatively slower motion. In order to guarantee a safe deployment, let's assume that the registration errors take the maximum value of $2.4 \mathrm{~mm}$ for both the starting and ending points in either direction (i.e., total error of $4.8 \mathrm{~mm}$ at each side), then omitting the outer parts, the diameter of the corridor drops to $4.2 \mathrm{~mm}$ in systole and $17.2 \mathrm{~mm}$ in diastole. Since the robot always follows the centerline of the corridor, any device with diameter less than $4.2 \mathrm{~mm}$ can be deployed safely within such a corridor. 
For all the 12 subjects, the simulated deployment of the virtual robot through dynamically registering the corridors with real-time MRI showed no collision with the inner boundaries of LV. The tested diameter of the robotic link was $4 \mathrm{~mm}$. As depicted in Fig. 5 after an initial user-defined idling period, there are two deployment phases.

Table 1. The registration errors for the updated corridors on the real-time slices $(n=12)$

\begin{tabular}{lllllll}
\hline Subject/Error $(\mathrm{mm})$ & \multicolumn{3}{l}{ End point $\left(\varepsilon_{\mathrm{E}}\right)$} & & \multicolumn{3}{l}{ Start point $\left(\varepsilon_{\mathrm{S}}\right)$} \\
\cline { 5 - 7 } 1 & Max & Min & Average & Max & Min & Average \\
2 & 1.9 & 1.6 & 1.8 & 1.6 & 1.2 & 1.5 \\
3 & 2.1 & 1.8 & 1.9 & 1.4 & 1.1 & 1.2 \\
4 & 2.4 & 2.2 & 2.3 & 1.5 & 1.1 & 1.2 \\
5 & 2.0 & 1.7 & 1.8 & 1.7 & 1.3 & 1.6 \\
6 & 2.1 & 1.9 & 2.0 & 1.5 & 1.2 & 1.4 \\
7 & 2.1 & 1.8 & 1.9 & 1.3 & 1.0 & 1.2 \\
8 & 2.2 & 1.8 & 2.0 & 1.4 & 1.1 & 1.3 \\
9 & 2.4 & 1.9 & 2.3 & 1.5 & 1.2 & 1.3 \\
10 & 2.0 & 1.7 & 1.8 & 1.6 & 1.1 & 1.4 \\
11 & 2.2 & 1.7 & 1.9 & 1.4 & 1.0 & 1.2 \\
12 & 2.2 & 2.0 & 2.1 & 1.2 & 1.0 & 1.1 \\
\hline
\end{tabular}

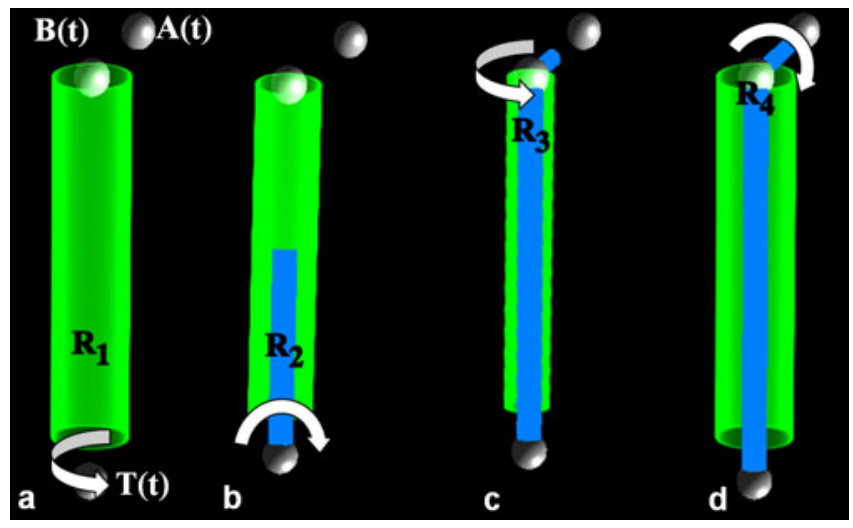

Fig. 5. Deployment is simulated (the images have the same field of view): (a) and (b) spans Phase I. Phase II starts at (c) and ends in (d).

The Phase I is the extension of the first link from the apical entrance toward the bending point, which entails the actuation of the first two rotational DOFs (i.e., $\mathrm{R}_{1}$ and $\mathrm{R}_{2}$ ) to maintain the deployed part inside the corridor. Once the distal end of the first link reaches near $\mathrm{B}(\mathrm{t})$, the Phase II starts with the extension of the second link toward the targeted aortic annulus. Concurrently, the third and fourth rotational DOFs (i.e., $\mathrm{R}_{3}$ and $\mathrm{R}_{4}$ ) are also actuated to maintain the second link along the aortic annulus midline. After reaching the target, the robot maneuvers to hold the position: the base of the robot at $\mathrm{T}(\mathrm{t})$, the robot inside the corridor, and the tip of the second link at $\mathrm{A}(\mathrm{t})$. 
It is noteworthy that the presented computational core is connected to the MR scanner via a local area network (LAN) for a two-way communication: (i) real-time data transfer from the scanner to the computational core (thereby achieving a refreshing rate of $20 \mathrm{fps}$ ) and (ii) in reverse on-the-fly adjustment of the imaging parameters from the control module of the core, as we have demonstrated before [8].

\section{Conclusions}

This paper introduces a novel computational methodology for planning and performing real-time MRI-guided interventions in a beating heart. In all our studies it was able to generate a dynamic cylindrical corridor and update it with real-time single-plane MRI. Future work includes testing it on an actuated cardiac phantom [9] and automatically tracking the aortic annulus centerline on LA real-time MRI.

Acknowledgments. Supported by the National Science Foundation (NSF) award CPS0932272. All opinions, findings, conclusions or recommendations in this work are those of the authors and do not necessarily reflect the views of our sponsors.

\section{References}

1. Jolesz, F.A.: Future perspectives for intraoperative MRI. Neurosurg. Clin. N. Am. 16, 201$213(2005)$

2. Li, M., Mazilu, D., Wood, B.J., Horvath, K.A., Kapoor, A.: A robotic assistant system for cardiac interventions under MRI guidance. In: Proc. SPIE 7625, pp. 76252X (2010)

3. McRae, M.E., Rodger, M., Bailey, B.A.: Transcatheter and transapical aortic valve replacement. Crit. Care Nurse 29, 22-37 (2009)

4. Horvath, K.A., Mazilu, D., Guttman, M., Zetts, A., Hunt, T., Li, M.: Midterm results of transapical aortic valve replacement via real-time magnetic resonance imaging guidance. J. Thorac. Cardiovasc. Surg. 139, 424-430 (2010)

5. Yeniaras, E., Deng, Z., Davies, M., Syed, M.A., Tsekos, N.V.: A Novel Virtual Reality Environment for Preoperative Planning and Simulation of Image Guided Intracardiac Surgeries with Robotic Manipulators. Stud. Health Technol. Inform. 163, 716-722 (2011)

6. Zhou, Y., Yeniaras, E., Tsiamyrtzis, P., Tsekos, N., Pavlidis, I.: Collaborative Tracking for MRI-Guided Robotic Intervention on the Beating Heart. In: Jiang, T., Navab, N., Pluim, J.P.W., Viergever, M.A. (eds.) MICCAI 2010. LNCS, vol. 6363, pp. 351-358. Springer, Heidelberg (2010)

7. Elgort, D.R., Wong, E.Y., Hillenbrand, C.M., Wacker, F.K., Lewin, J.S., Duerk, J.L.: Realtime catheter tracking and adaptive imaging. J. Magn. Reson. Imaging 18, 621-626 (2003)

8. Christoforou, E., Akbudak, E., Ozcan, A., Karanikolas, M., Tsekos, N.V.: Performance of interventions with manipulator-driven real-time MR guidance: implementation and initial in vitro tests. Magn. Reson. Imaging 25, 69-77 (2007)

9. Sternberg, N.V., Hedayati, Y., Yeniaras, E., Christoforou, E., Tsekos, N.V.: Design of an actuated phantom to mimic the motion of cardiac landmarks for the study of image-guided intracardiac interventions. In: ROBIO, pp. 856-861 (2010) 